\title{
Getting Started in Clinical Research
}

\author{
James W. Fleshman, Jr., M.D. ${ }^{1}$
}

ABSTRACT

Clinical research is an important part of an academic surgery practice. To be successful, it is important to understand the multiple regularity committees and organizations that impact research. The author briefly reviews these groups and provides guidance on how to initiate and conduct research.

KEYWORDS: Colon and rectal surgery, clinical research

Objectives: Upon completion of this article, the reader should be familiar with clinical research in colon and rectal surgery.

$I_{t}$ is one of the great pleasures of academic surgery to be able to participate in clinical research. Most patients who come to the academic institution are willing to participate in meaningful studies that potentially could change the practice of medicine and improve outcomes for patients. As an academic surgeon, the privilege of taking care of patients involved in research is one of the highest benefits and honors that can be bestowed on a physician. My career at Washington University School of Medicine in St. Louis has provided me with such an honor and gift. Throughout my career, the focus of my research has transitioned from basic science to translational to clinical research. It is one of the most exciting things that I do on a daily basis and allows me to continually reevaluate the way I take care of patients, train residents, and relate to other medical care providers.

When starting clinical research, there are certain facts that are very important for the clinical research physician. I call this getting to know your "regulatory alphabet." The Institutional Review Board (IRB) or the Human Studies Review Committee (HSRC) oversees the institutional clinical research. This body can be your friend or your worst nightmare depending on whether you follow the rules. The Human Research Protection Office (HRPO) audits and mandates professionalism within clinical research. This office is a governmental agency and uses the Office of the Inspector General (OIG) as its enforcement arm. OIG agents carry guns and badges and can bring criminal charges. The HRPO protects patients from fraud and improper clinical research and lends legitimacy to the clinical research being performed by academic institutions.

All clinical research must be in compliance with the Health Insurance Portability and Accountability Act (HIPAA). The legislative arm of the government has mandated that we should protect the confidential, sensitive information of each patient, even while performing clinical research. For this reason, HIPAA has set up guidelines which we follow to avoid identification of patients in publications and reports of the research.

Particular types of research provide their own institutional oversight. For example, if one practices in a comprehensive cancer center, the research is coordinated through the Peer Review Monitoring Committee (PRMC). This is a conflict prevention and management program by which researchers can discuss and review potential studies to limit overlap, prevent encroachment
${ }^{1}$ Section of Colon and Rectal Surgery, Washington University School of Medicine, St. Louis, Missouri.

Address for correspondence and reprint requests: James W. Fleshman, Jr., M.D., Colon and Rectal Surgery, Washington University School of Medicine, Queeny Tower, \#14102, 660 S. Euclid Ave., Campus Box 8109, St. Louis, MO 63110 (e-mail: fleshmanj@wustl. edu).
Career Development; Guest Editor, David E. Beck, M.D.

Clin Colon Rectal Surg 2011;24:100-105. Copyright (C) 2011 by Thieme Medical Publishers, Inc., 333 Seventh Avenue, New York, NY 10001, USA. Tel: +1(212) 584-4662.

DOI: http://dx.doi.org/10.1055/s-0031-1278405.

ISSN 1531-0043. 
by a proposed study upon a patient population that is being used for an established study, and expose and resolve conflict between members of the clinical trials group.

Each institution requires that a Data Safety Monitoring Board (DSMB) audit the collected data for all intrainstitutional trials. These are trials that are investigator initiated and are not being monitored by outside groups, such as industry or government. The DSMB can close clinical research just as easily as the HRPO, IRB, and other groups already mentioned. It is important that the DSMB be involved to avoid such fiascos as have occurred in recent experience in the military; data falsified and outcomes invented to prove an investigator's point. Therefore, the DSMB prevents any question of the legitimacy of the data that is produced.

The Institutional Billing Matrix (IBM) has been established to eliminate fraud by clinical researchers receiving double payment on research patients. The institution must keep track of all patients who are involved in clinical trials. In some trials, the investigator is being reimbursed for diagnostic procedures. Most patients participating in industry or government led trials will have funds directed toward paying for some of their care. The institution billing matrix should alert the clinical researcher and hospital of the potential for double billing for patient care. This occasionally occurs without the clinical researcher's knowledge. Even so, the researcher is responsible for billing fraud, a criminal offense.

\section{GETTING STARTED}

First, identify a question and develop a hypothesis before designing a trial. This is most often observation driven, but now it may be driven by a new technology or the desire to evaluate comparative effectiveness.

Next, identify an appropriate study population. Basic science can answer some questions for clinical research; however, the majority of clinical research is performed on patients. The appropriate patient base is important. In some circumstances, such as new technology, the first step may be an animal study to provide safety and efficacy data prior to introduction into the patient population. The study population may not be patients. There is a role for studying the educational process in surgery within or after residency training programs or as it affects assimilation of new technology.

Finally, all clinical research should incorporate a control group for comparison. Retrospective or prospective series of patient events or outcomes are no longer adequate to establish a new intervention or change clinical practice. The control group can be current (as with randomized controlled trials) or consecutive, but not involved in the study directly (as with case-matched controlled trials). Historical controls are the least accu- rate method of establishing a control, and time has made them irrelevant because there are other confounding factors involved in their care, such as advances in new technology unrelated to the technology or the treatment being evaluated.

\section{PRESTUDY STATISTICS}

A power calculation is essential to determine the appropriate sample size for both a prospective comparison and a retrospective review comparison. This requires an estimation of the expected difference between two study groups or a study group and the control. The appropriate comparative statistical method can be used in a power calculation formula to determine the number of patients needed in the study. The sample size calculation is based on achieving significance ( $P$-value) given the predicted percent difference between the two groups at a desired power (percent level of confidence). This is available in most commercial statistical packages or through the biostatistics core of an academic institution.

Before starting a comparative trial, the untoward events (such as complications or unanticipated consequences) that would require study closure must be defined. "Stopping parameters" for new techniques or drugs prevent study completion with harm to a significant number of patients. This is obviously not needed for retrospective reviews.

It is helpful to design a study in collaboration with a statistician. Most institutions have a "central core" of biostatistics, which is available on a consultation basis. The participating statistician would be reimbursed for time spent or as a percentage of effort on the trial itself.

\section{DATABASE STUDIES}

One of the most basic forms of clinical research is the retrospective or prospective review of patients. It is important to design the database to be as comprehensive as possible; however, resources may restrict the ability to enter massive amounts of data to a comprehensive database. The estimated salary outlay for a database manager is approximately $\$ 35,000$ per year for a full-time manager. It is possible to incorporate other functions into the database manager's job description, such as most institutions require design and production of clinical reports or troubleshooting for office computing needs.

Most institutions require IRB approval before the first patient is entered into a database. The IRB has now become the overall protector of all patient data being used in research, and databases are now scrutinized by the IRB and must be approved before beginning the first evaluation of a patient in a retrospective study.

Search and report packages should be built into the database. This gives the section or practice an update on patient volumes and patient diseases that can help 
design trials around specific questions. If the database manager is not capable of designing these packages, the researcher should consider contracting with the institution information systems to maintain, update, and backup the database and design the reports. A simple package of reports, as a start, can be eventually uploaded into a more sophisticated, analytic package for the future.

\section{LEVEL OF EVIDENCE}

The American Society of Colon and Rectal Surgeons (ASCRS) has designated different levels of evidence for use by its Practice Parameters Group. A "level of evidence" appropriate for writing a parameter may not be possible for a small clinical trial effort to achieve. As a researcher gains more experience, the "level of evidence" produced by the institution trial should improve. To establish a clinical trials mechanism in your own institution, the accompanying listing of levels of evidence may be most appropriate (refer to Table 1). The highest level (I) of evidence is a systematic review of multiple, similarly designed randomized controlled trials with a large number of patients in each trial. Strong recommendations for patient care can be made from these reviews. The systematic review process has been formalized by the Cochrane Review Group, but can be performed on an individual basis. The criteria followed for the systematic review including the type of study, the number of patients enrolled, the analysis undertaken, and the controls provided should be carefully documented. With this type of review, much larger numbers of patients are able to be collated to evaluate the question being asked.

The randomized controlled trial (RCT) is the highest level of evidence (level II) that a single institution or a single group of researchers can produce around a particular question. The RCT must have predetermined parameters for inclusion and exclusion, a sample size calculation for desired power and significance, and a regulatory process by which the entry of patients into the trial are monitored and the standardization of the procedure or process is achieved.

There are circumstances or institutions where randomized controlled trials cannot be performed. A case-matched control trial (CMCT) based on prospective

\section{Table 1 Levels of Evidence}
I. Systematic Review of Randomized Controlled Trials
II. Randomized Controlled Trial
III. Case Matched Controlled Trial - Prospective or Retrospective
IV. Prospective Series
Database
Retrospective Series

or retrospective data collection is the next best alternative (level III) to achieve an answer for a clinical question. The case-matched control mechanism requires that multiple factors, which may or may not be related to the question being asked, be established to select the controls and compare with the prospective group of patients being enrolled. For example, in patients undergoing colectomy, the laparoscopic approach can be compared with the open approach based on selection by gender, age, disease process, medical severity index scores, body mass index (BMI), and stage of disease. Simply by standardizing these parameters, the prospective collected group can be compared with the retrospective evaluated group to provide a more homogeneous population upon which to perform statistical analysis.

The final level of evidence derived from a database evaluation of a prospective or a retrospective series provides only description statistics (level IV). The conclusion may be enhanced by performing a populationtype study. Extremely large numbers derived from national databases can at times be considered high-powered evidence. However, the circumstances must be carefully controlled and one must be careful that selection bias, heterogeneity of the population, and outside influencing factors do not undermine the power of the analysis itself.

\section{COLLABORATION POSSIBILITIES}

Young investigators have numerous possibilities for getting started in clinical research. The first possibility is to establish a working group of investigators, with whom you are familiar, to combine database information, or to query national databases around a particular clinical question. It is possible to develop a consortium for establishing prospective studies around topics such as new technology, rare conditions, resident education, and expensive technology. In the past, clinical trials were almost exclusively run through the Cooperative Trials Groups for studying cancer. Now numerous trial groups are receptive to new members to look at issues specifically around the topic for which the trial group was established. The most frequently thought of Cooperative Trials Group subject is cancer. The American College of Surgeons Oncology Group, the Cancer and Lymphoma Group B, the National Surgical Adjuvant Bowel and Breast Project, the Eastern Cooperative Oncology Group, the North Central Cancer Trials Group, Southwest Oncology Group, and Radiation Therapy Oncology Group are all examples of groups that study cancer. It is easy to belong to one of these groups if your institution has an existing relationship or has the core resource backup needed to obtain membership. Individual private practitioners can belong as accruing members. These groups provide education and have yearly meetings at which they encourage participation in their 
established trials. Each member has the freedom to submit a potential protocol to the overall group regarding a specific question. The group as a whole will evaluate the protocol and may accept it as a protocol. The trial group must be willing to expend the funds given to it by the government (National Institutes of Health $[\mathrm{NIH}])$ to answer the question.

Industry has become a major resource for sponsoring small clinical trials. Some are industry initiated, and the sponsor selects relevant investigators around their topic. Others can be initiated by an investigator or group of investigators. The industry sponsor can decide to provide funds for a multicenter trial adequate to answer a specific question. New technology has been evaluated in this way over the last two decades. This approach works well as long as the investigators remember the potential for bias from the industry partner. It is an important mechanism because governmental funds are rarely directed to evaluating new technology, new drugs, or implants in our patients.

Finally, the ASCRS has become a resource for young investigators. There are requests for proposals (RFPs) from the ASCRS Research Foundation for projects addressing the top 20 research topics revealed through a Delphi project. ${ }^{1}$ A new committee of the ASCRS, called the Research Development Committee headed by Dr. Walter Koltun, is designed to use the committee members, most of whom are young investigators, to begin the multicenter process of establishing projects that are important to our patients and practice. The ASCRS Foundation has also begun funding K23 (clinical) and $\mathrm{KO} 8$ (basic) projects through the NCI around the area of colon and rectal cancer. This mechanism requires a young investigator to dedicate $75 \%$ of his or her effort on a 2- to 5-year project. This time is covered by a combination of funds from the government and the ASCRS. It is important for our young members and upcoming researchers to understand these mechanisms for obtaining funding and support.

\section{GETTING STARTED IN THE CLINICAL TRIAL MECHANISM}

Developing a clinical trial mechanism de novo at an institution involves several key steps. Step I is to identify a potential project and work through the process previously stated to develop a hypothesis and the best method of answering this question. Step II is to hire a clinical trials coordinator or clinical research assistant (CRA) to coordinate the support services of the clinical trial. These individuals collect data, collate data, organize data, enter data, and solicit, enroll, and follow patients. Most of these individuals start at a salary of $\$ 35,000$ and increase according to the responsibilities and the volume of patients enrolled in trials. The goal should be for the coordinator to become self-supporting through the trial mechanism as studies are funded and money is designated for coordinator support.

The Contracts Committee for the institution needs to approve participation in all outside funded clinical trials, especially those sponsored by the industry or government. Outside funding requires monitoring, and the Contracts Committee is the oversight body for making sure the funds are used appropriately.

Once the protocol is defined, determining the cost per patient is extremely important. This involves not only a calculation of the time that the clinical trial coordinator will invest but also the investigator's time, the cost of any laboratory testing, and any materials that are involved in the procedure. The IRB fee at most institutions is approximately $\$ 3,000$. It is important to remember to assign this to the industry sponsor. The percent effort for each trial for a clinical trial coordinator should be somewhere between 10 to $15 \%$. The principal investigator effort is probably less than $5 \%$ for a clinical trial in which the majority of effort will be made by the CRA. The usual calculated cost per patient enrolled into a clinical trial with long-term follow-up is close to $\$ 4,000$ per patient. The Contracts Committee will also negotiate institutional indirect costs to be added to the contract. This figure can be as high as $50 \%$ of the direct costs. This places quite a burden on the investigator seeking industry sponsorship. However, it supports the hidden overhead for large institutions.

The next step would then be to submit the protocol and a stylized consent form to the IRB. The IRB consent should be written in eighth-grade language and easily understood by any individual. The protocol should be basic yet detailed enough to prevent numerous questions being returned to the investigator from the IRB. It is a long process, even when everything goes well; but if multiple queries are returned to the investigator, the IRB process can be drawn out to many months.

Outside funding requires that the billing matrix be notified to make them aware of potential conflicts in reimbursement for the study. This is a simple matter but involves more paper work for which the CRA should be responsible.

It is important to educate the office staff and the collaborating faculty of the inclusion/exclusion criteria and the basic outline on all active trials to make sure there is a cooperative effort to enroll patients. It is impossible for any individual investigator to develop an adequate volume and enrollment to reach the numbers required to achieve significance in a clinical trial question. Successful accrual can be predicted by determining the number of patients entering the practice with the condition of interest, dividing by two for those that will meet inclusion criteria of the study and dividing again by two to achieve the number that will consent to participate.

A secured area is needed for maintaining clinical trial report forms or CRFs. This means firewalls for the 
computer program, a locked room for storing notebooks, and limited access from other nonclinical trials-related personnel. At all times, the files and clinical report forms should be maintained as ready for an audit. There are several sources of audit that can put the investigation and the clinical trials process as risk. As mentioned earlier, the Data Safety Management Board (DSMB) of the institution, the IRB, the Office of Investigator General (OIG), the FDA, the industry sponsor, the clinical trials group (such as ACOSOG) can ask to audit the data which is being collected. The usual lead time notice for this audit is 2 weeks. In a fairly large study, the amount of time involved to clean the case report forms to achieve the audit readiness is much greater than the time from notification to audit.

\section{OBTAINING IRB APPROVAL}

As mentioned earlier, the key to successful IRB submission is to summarize your project so that a nonmedical person can understand the project, i.e., think like an eighth grader. Decision to submit research for IRB approval is fairly simple in today's research atmosphere. Any research that would be eventually published should receive IRB approval. There are several levels of IRB submission, which include expedited, routine, and extended or high-risk. The expedited group includes retrospective reviews of de-identified data from IRBapproved databases, survey of national organizational members, or the number of times the elevator sticks on your floor in the hospital. Routine refers to randomized controlled trials, case-matched prospective controlled trials, industry sponsored drug study, and comparison of surgical techniques using either randomized controlled trials or case-matched control reviews.

Extended or high-risk submissions include subjects such as genetic profiling, HIV (human immunodeficiency virus) population, pediatrics, studies in which patient data may be identifiable, and experimental highrisk drug studies. The category designation refers to the amount of effort that would be required to obtain IRB approval and, as a result, the number of pages in the IRB submission. Table 2 refers to the information that you will need to successfully achieve IRB approval. These categories may occur in a different order at different institutions, but the basic grouping is the same. The IRB in most institutions has an online submission process and every blank should be filled to accommodate their needs and to be successfully reviewed. One should automatically assume that the first submission will not be successful and that the IRB will return your submission with questions, a request for more detail and/or preliminary data. The newly graduated, first-time, submission investigator should not be discouraged or take any of these "returns to sender" as personal. It happens to all of us, even after many years of doing clinical research. It is part
Table 2 Institutional Review Board (IRB) Submission: What You Need to Start
1. Demographics
2. Research team
3. Source(s) of support
4. Waiver of consent
5. Other institutional reviews/requirements
6. Participants
7. Basic project information
8. Drugs/devices
9. Genetic research
10. Recruitment and consent
11. Methods
12. Risks
13. Benefits
14. Privacy and confidentiality
15. Data analysis
16. Future research

of the process. If the process is still confusing to the new investigator, one suggestion may be to apply for membership on your local IRB so that you become more familiar with the process and thereby take away the unknown and the secrecy that is presumed to be part of the process.

\section{ESSENTIALS FOR DEVELOPING A RESEARCH-FOCUSED SECTION}

As mentioned earlier, the first steps are to hire a database manager, establish a database, and hire a clinical trials coordinator. This initial outlay of money should be something that each new investigator requests from the department during the hiring process. Although almost all clinical trials research funding comes from operations and funding derived from the clinical trial itself, the initial startup cost is something that most departments should be able to accommodate and would be most helpful to any young investigator.

Make the IRB submission easy by learning the process, either by joining the team or spending time looking at the electronic version. Hire someone to manage the paperwork, such as a secretary or your clinical trials coordinator and think like an eighth grader.

Each new investigator will have a blank slate. As a new surgeon begins operating, keeping an easy erase board in the office to keep a list of projects for clinical trials, retrospective review questions, or basic science questions may be helpful to remind the new investigator that there are still unknown areas around the area of colon and rectal surgery. These projects can be checked off as the trials are developed. Residents, fellows, and other investigators sometimes utilize the project list as starting points for their own projects and would obviously 
include the generator of the idea in the collaborative effort.

It is important to make contacts for collaborative projects. This would include establishing groups of researchers based on like-minded fellows from the fellowship class from which you recently graduated or the ASCRS Research Foundation Grant Review Group, of which Dr. Garcia-Aguilar is the president. Introduction to industry representatives in the marketing or research and development groups can open doors to participating in comparative effectiveness research or establishing safety for new technology. Institutional or departmental research groups, such as the PRMC, IRB, or DSMB will be populated by institutional researchers. Getting to know these individuals can open doors for participation, collaboration, and guidance mentoring from senior investigators looking for new ideas and enthusiastic individuals willing to work. It may be necessary to join a cooperative group, and the institution may have to pay money for membership. In most circumstances, a university will already belong to most of these cooperative trial groups. If so, the new investigator should ask to attend the cooperative trial group meeting to learn more about establishing a clinical trials research program within colon and rectal surgery.

Publishing background data around the area of interest by submitting a review article or a retrospective background data review is appropriate at the beginning of a career. Prospective series with case control usually comes with more operative experience. Early on, this may be possible if the investigator is in a well-established group with several active senior members willing to share their patient data. Once the investigator has become well established, then a randomized controlled trial or phase two study process may become possible and important outcomes will be available for publication and treatment changing conclusions.

Once the individual institution and investigator have become established and data has been published, it is important to publish multiinstitutional data to confirm the single institutional data. The studies should also be able to generalize the findings to community practice. It is important that an investigator look critically at the results of recently completed trials to identify unanswered or newly revealed questions. This sets up the process to be self-perpetuating. Any good study will have numerous questions and may only have one answer. The questions are probably the most important product of the completed research from an investigator point of view.

It is important as you begin your pathway into clinical research that the regulatory and mechanistic issues not become onerous and act as anchors to your progress. Remember that clinical trials are one of the most rewarding parts of being an academic physician. Above all, as you design trials, collect data, and publish reports, it is important to remember to enjoy yourself and enjoy the fact that clinical research will inevitably have an impact on your patient's care. Good luck.

\section{DISCLOSURE}

The author is an academic surgeon at Washington University, St. Louis, Missouri.

\section{REFERENCE}

1. Burt CG, Cima RR, et al. Developing a research agenda for the American Society of Colon and Rectal Surgeons: results of a Delphi approach. Dis Colon Rectum 2009;52(5):898-905 